\title{
Tratamento cirúrgico da comunicação buco-sinusal ocorrida durante a exodontia para reabilitação com prótese dentária: relato de caso
}

\author{
Surgical treatment of oral-sinus communication during dental extraction for dental \\ rehabilitation: case report
}

Tratamiento quirúrgico de la comunicación del seno oral durante la extracción dental para rehabilitación dental: reporte de caso

Joyce Meneses Machado Silva ${ }^{1}$, Renata da Silva Pereira ${ }^{1}$, Leonara Severiano e Silva ${ }^{1}$, Weizia Gomes da Rocha ${ }^{1}$, Wanderley Barros dos Santos ${ }^{1 *}$, Waldir Martins Veloso Soares ${ }^{1}$, Carlos Sousa Mello de Almeida ${ }^{1}$, Carlos Eduardo Campos Ramos ${ }^{1}$, Laís Regina de Oliveira Cavalcanti ${ }^{1}$, Tayguara Cerqueira Cavalcanti ${ }^{1}$.

\section{RESUMO}

Objetivo: Apresentar o relato de um caso de comunicação buco-sinusal tratada pela técnica do retalho bucal vestibular ocorrida durante a realização de cirurgia pré-protética. Detalhamento do caso: Foi realizada a exodontia dos elementos remanescentes da maxila que culminou com a comunicação indevida do seio maxilar com o meio bucal, no qual foi realizada, durante o mesmo ato cirúrgico, a confecção de um retalho vestibular para o fechamento desta comunicação. Discussão: As comunicações buco-sinusais estão relacionadas à intercorrências após exodontias de dentes que apresentam as raízes com íntima relação com a parede sinusal, dessa forma os segundos molares superiores são mais acometidos, por suas raízes terem maior contato com o assoalho do seio maxilar. Considerações finais: A comunicação buco-sinusal trata-se de uma complicação relativamente frequente na área odontológica. Para o seu tratamento devem ser levados em consideração alguns parâmetros relacionados ao seio maxilar com os demais dentes, visando o correto diagnóstico para obter o melhor prognóstico para o paciente.

Palavras chaves: Fístula bucal, Seio maxilar, Cirurgia.

\begin{abstract}
Objective: To present or report a case of oral-sinus communication treated by the buccal vestibular flap technique that occurred during a pre-prosthetic surgery. Case Detail: Extraction of the other elements of the maxilla was performed, resulting in inadequate communication of the maxillary sinus with the mouth, performed during the same surgical procedure, in the preparation of a vestibular flap to close this communication. Discussion: Buco-sinus communications are related to complications after tooth extractions with roots that are closely related to the sinus wall, thus the upper second molars are more affected because their roots have greater contact with the maxillary sinus floor. Final considerations: Oral-sinus communication is a relatively frequent complication in the dental field. For its treatment, some parameters related to the maxillary sinus with the other teeth must be taken into consideration in order to obtain the correct diagnosis to obtain the best prognosis for the patient.
\end{abstract}

Keywords: Buccal fistula, Maxillary sinus, Surgery.

${ }^{1}$ Centro Universitário CESMAC, Maceió-Alagoas. * E-mail: Wanderley.barros108@gmail.com

SUBMETIDO EM: 10/2019

ACEITO EM: 11/2019

PUBLICADO EM: 1/2020

REAS/EJCH | Vol.Sup.n.39 | e2127 | DOI: https://doi.org/10.25248/reas.e2127.2020 Página 1 de 7 


\section{RESUMEN}

Objetivo: Presentar o informar un caso de comunicación de seno oral tratado con la técnica de colgajo vestibular vestibular que ocurrió durante una cirugía pre-protésica. Detalle del caso: Se realizó la extracción de los elementos restantes del maxilar, que culminó en la comunicación inadecuada del seno maxilar con mi boca, que se realizó durante el mismo procedimiento quirúrgico, la preparación de un colgajo vestibular para cerrar esta comunicación. Discusión: La comunicación buco-sinusal está relacionada con complicaciones después de las extracciones de dientes con raíces estrechamente relacionadas con la pared del seno, por lo que los segundos molares superiores están más afectados porque sus raíces tienen un mayor contacto con el piso del seno maxilar. Consideraciones finales: La comunicación oral-sinusal es una complicación relativamente frecuente en el área dental. Para su tratamiento, se deben tener en cuenta algunos parámetros relacionados con el seno maxilar con los otros dientes, con el objetivo de obtener el diagnóstico correcto para obtener el mejor pronóstico para el paciente.

Palabras claves: Fístula bucal, Seno maxilar, Cirugía.

\section{INTRODUÇÃO}

A comunicação buco-sinusal indica o acesso anormal entre o seio maxilar e a cavidade bucal. Essas conexões abertas acontecem frequentemente durante extrações dentárias, quando o ápice do dente apresenta uma íntima relação com a cavidade do seio maxilar, sendo complicações cirúrgicas relativamente frequentes na odontologia (NOGUEIRA EF, et al., 2018; PATEL R, et al., 2019). A proximidade das raízes de pré-molares e molares superiores com o seio maxilar é uma das principais causas dessa comunicação, nos quais, os segundos molares, por suas raízes terem maior proximidade com o assoalho do seio maxilar, são os dentes mais citados na literatura (CUNHA G, et al ., 2017; NOGUEIRA EF, et al., 2018).

As comunicações buco-sinusais apresentam prevalência em homens, na faixa etária de aproximadamente 40 anos de idade e ocorre por manobras iatrogênicas, trauma, cirurgia para remoção de cistos e enucleação de tumores, patologias ósseas, colocação de implantes e cirurgias mais complexas como a ortognática. Após a comunicação buco-sinusal, se não houver regressão, poderá tornar-se crônica e formar uma fístula bucosinusal, que por sua vez, causará escoamento dos líquidos da boca para a cavidade nasal, distúrbios de fala, respiração, dor, epistaxe e sinusite. Sánchez e colaboradores relataram que $50 \%$ dos pacientes desenvolvem sinusite após 48 horas, enquanto 90\% o fazem após duas semanas (SANCHEZ AS, et al., 2018; NOGUEIRA EF, et al., 2018).

O diagnóstico das comunicações buco-sinusais é realizado com o auxílio de procedimentos clínicos e radiográficos, os quais apresentam caráter decisivo na conduta terapêutica adequada. Nos exames radiográficos, as radiografias periapicais, evidenciam a descontinuidade da linha radiopaca que delimita 0 assoalho do seio maxilar, por outro lado, as projeções extra-orais, como a radiografia panorâmica e a incidência de Waters, são limitadas com relação às comunicações pequenas, tendo sua grande importância na observação do seio envolvido, que poderá apresentar uma radiopacidade difusa, quando comparada ao seio do lado oposto. A tomografia computadorizada fornece informações como tamanho da comunicação, características do osso e da lesão, além de não sofrer sobreposição, ultimamente vem sendo um recurso diagnóstico muito solicitado no planejamento das intervenções cirúrgicas, tanto pela fidelidade de informações quanto pelo custo benefício (COELHO JV e TORRES SG, 2018; VERAS FR, 2015).

No exame físico, a importância da Manobra de Vasalva é indiscutível, e deve ser realizada em todas as exodontias em região posterior da maxila, sendo extremamente valorosa no diagnóstico da comunicação buco sinusal. A manobra consiste em pressionar as asas nasais bilateralmente, obstruindo as narinas do paciente e solicita que ele que expire o ar pelo nariz, mantendo a boca aberta. Se houver comunicação, o ar expirado sairá através do alvéolo, para o interior da cavidade bucal, provocando ruído característico de borbulhamento do sangue, acumulado no alvéolo dentário (PARISE GK e TASSARA LF, 2016). 
A conduta sobre o tratamento que as comunicações buco-sinusais terão será baseada no tamanho, que varia de acordo com o diâmetro da comunicação, sendo classificado como pequena moderada ou de grandes proporções, no tempo decorrido desde que foi diagnosticado e se houver uma infecção, bem como o tratamento adjuvante com analgésicos, anti-inflamatórios, antibioticoterapia adequada e descongestionante nasal (ROSA CB, et al., 2019).

O objetivo do presente trabalho é relatar um caso de comunicação buco-sinusal ocorrida durante a cirurgia pré-protética tratada pela técnica do retalho bucal vestibular.

\section{DETALHAMENTO DO CASO}

Paciente P.C.O, gênero masculino, 66 anos e feoderma, buscou atendimento na Clínica Odontológica do Centro Universitário Cesmac. Durante a anamnese relatou como queixa principal "Preciso de uma chapa nova". $\mathrm{Na}$ história da doença atual, o paciente afirmou ser portador de diabetes tipo II, controlado. No exame extrabucal não houve alterações de normalidade. No exame físico intrabucal foram observadas algumas ausências dentárias na maxila e mandíbula, presença de prótese parcial superior e inferior, além de apresentar mobilidade grau I nos dentes 26 e 27, tendo como diagnóstico final periodontite generalizada, Estágio IV Grau B (Figura 1).

Figura 1 - Aspecto inicial intraoral, mostrando as ausências dentárias no arco superior e inferior.

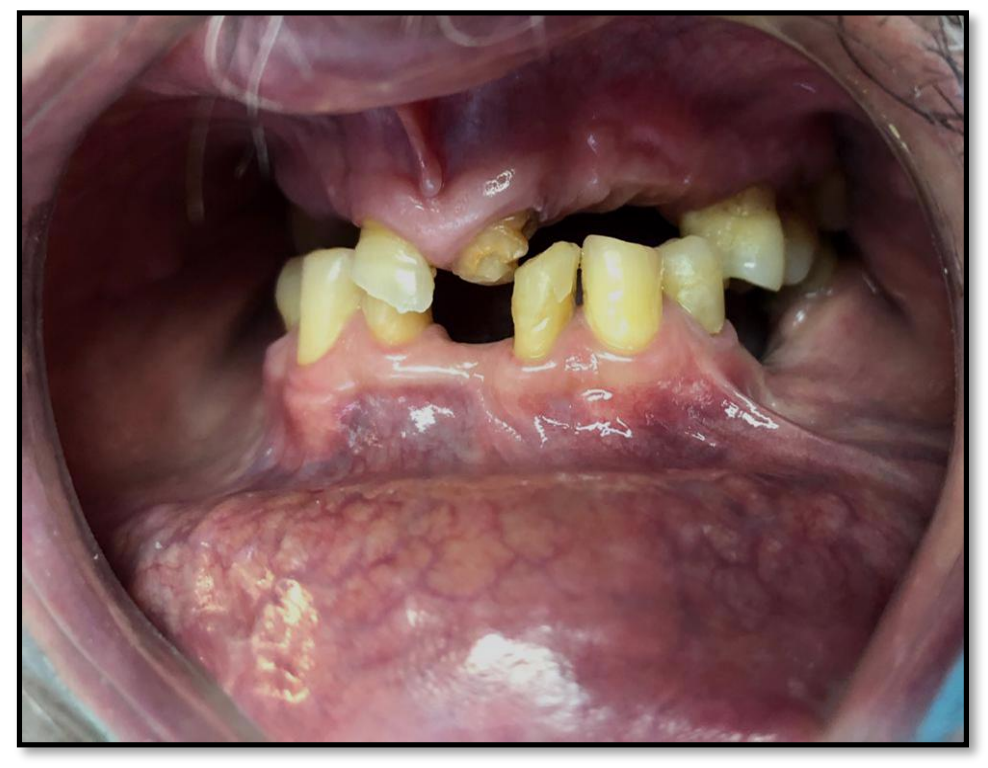

Fonte: Silva JMM, et al., 2019.

A conduta clínica foi realizada por meio do planejamento cirúrgico e protético através da confecção de uma prótese total imediata, para ser instalada após as exodontias dos dentes 17, 11, 21, 26 e 27, remanescentes da maxila.

Foi realizada a antissepsia intra e extrabucal com Clorexidina $0,12 \%$ e lodopovidina (PVP-I) $10 \%$, respectivamente, em seguida foi feito o bloqueio anestésico dos nervos alveolares superiores posteriores, médios e anteriores, de ambos os lados, além do nervo nasopalatino e palatinos bilateralmente, com Lidocaína $2 \%$ + Epinefrina 1.100.000. Durante as múltiplas exodontias, ocorreu a fratura da raiz palatina do dente 17, necessitando de osteotomia para remoção da mesma, resultando em uma comunicação com o seio maxilar (Figura 2). A comunicação buco-sinusal foi confirmada através da manobra de Vasalva, no qual o paciente emitiu forçadamente $o$ ar contra o nariz obstruído e a boca aberta. 
Figura 2 - Comunicação buco-sinusal após a exodontia do dente 17.

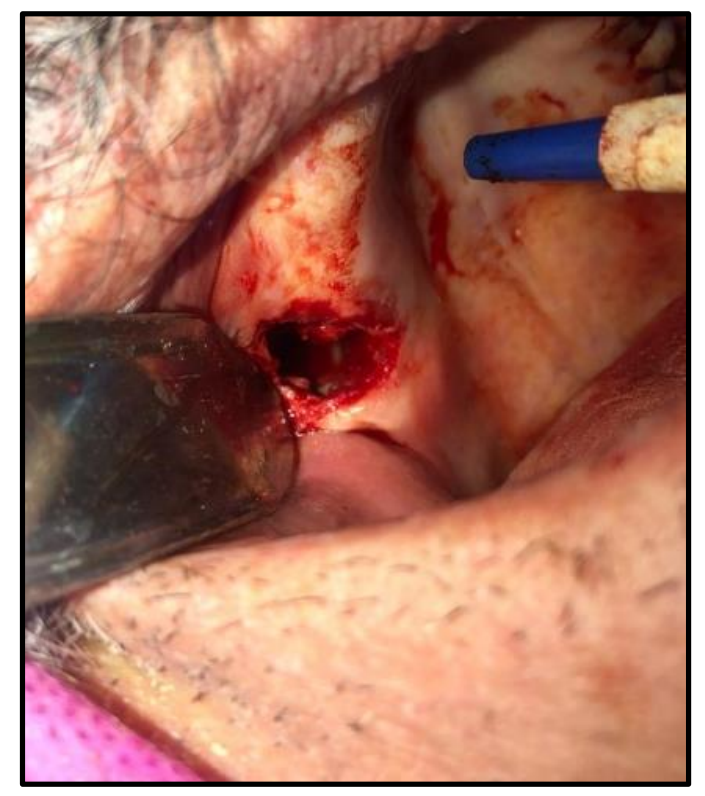

Fonte: Silva JMM, et al., 2019.

O tratamento proposto e executado foi o fechamento da comunicação buco-sinusal, realizado pela técnica do retalho vestibular, realizada por meio de duas incisões diagonais divergentes, em sentido superior, na porção vestibular do processo alveolar que correspondia desde a região do túber até a região do dente 17. Dessa forma, o tecido mole (mucosa, músculo e periósteo) foi descolado do osso, após a elevação do retalho uma incisão do periósteo foi realizada para melhor mobilidade do tecido mucoso e muscular até que este, por sua vez, atingisse o tecido gengival da porção palatina sem qualquer tensão no retalho e por fim, realizada a sutura dos tecidos de maneira passiva. (Figura 3 A e 3B).

Figura 3 - A, Retalho vestibular; B, Tracionamento do retalho vestibular para fechar a comunicação bucosinusal.

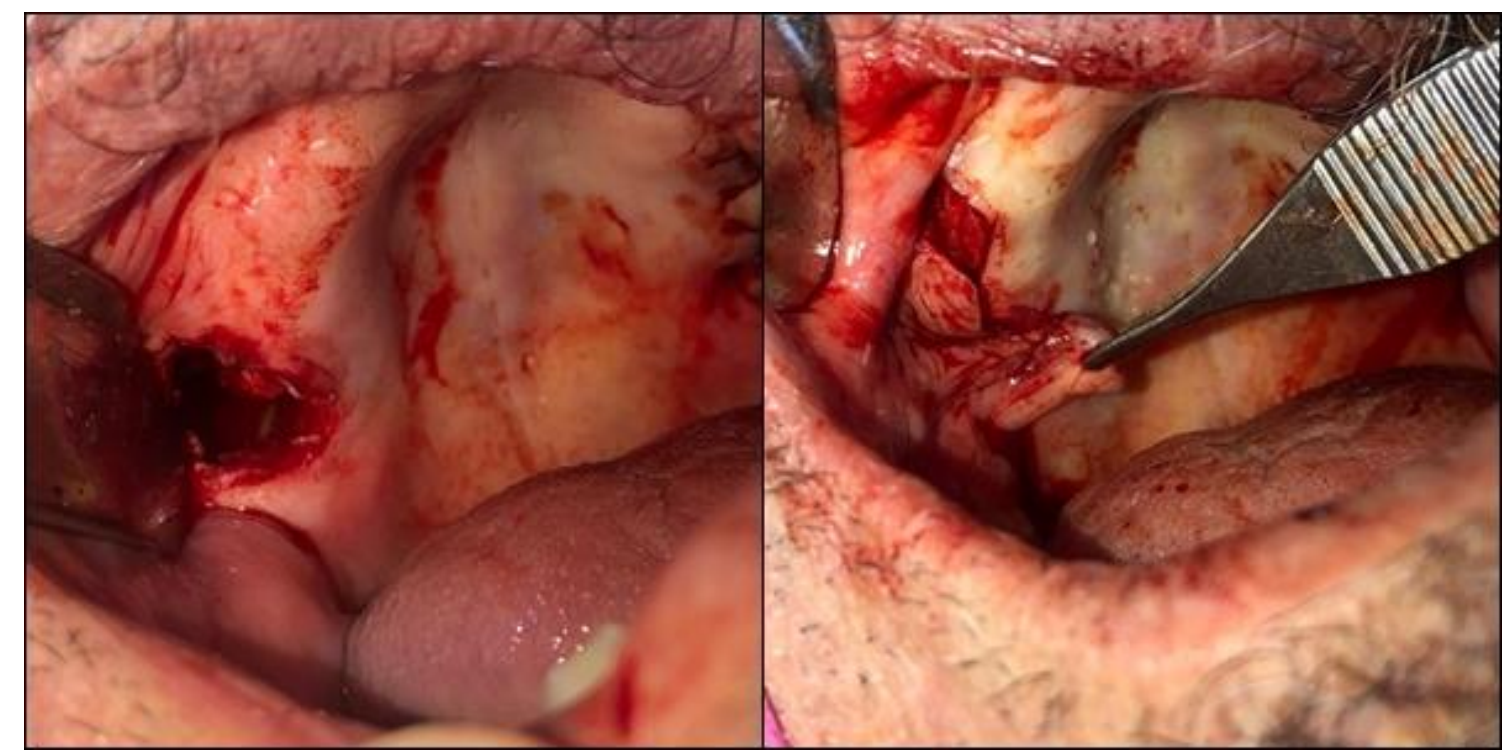

Fonte: Silva JMM, et al., 2019. 
A prótese total imediata foi instalada na mesma sessão para reabilitação funcional e estética. Foram prescritos os seguintes medicamentos: Amoxicilina $500 \mathrm{mg}$ de $8 / 8$ horas durante 5 dias, Paracetamol $500 \mathrm{mg}$ de 6/6 horas durante 2 dias e Diclofenaco de Sódio $50 \mathrm{mg}$ de $8 / 8$ horas durante 2 dias. A prescrição de analgésicos fez-se necessária para o controle da sintomatologia dolorosa, à antibioticoterapia torna-se efetiva no combate das infecções e da sinusite de origem odontogênica e o antinflamatório é capaz de reduzir a dor e o edema no pós-operatório.

No pós-operatório, o paciente foi orientado que em um período de 10 a 14 dias espirrasse com a boca aberta, evitasse assoar o nariz para não ocasionar uma pressão negativa ou positiva no interior do seio maxilar, assim como não utilizar canudos ao ingerir líquidos. Foi recomendado evitar bochechos por 24 horas, para não ocasionar desorganização do coágulo formado. Tais instruções realizadas em conjunto de uma boa higiene oral.

O paciente retornou após $24 \mathrm{~h}$ para realizar ajustes e controle da prótese e avaliação cirúrgica. No acompanhamento de 15 dias para avaliação e remoção da sutura local, observou-se a ausência da comunicação buco-sinusal e houve evolução favorável na cicatrização, com a presença de um tecido de granulação denso e a epitelização completa foi observada em três semanas com contração da ferida. (Figura 4).

Figura 4 - Cicatrização após 15 dias do procedimento cirúrgico.

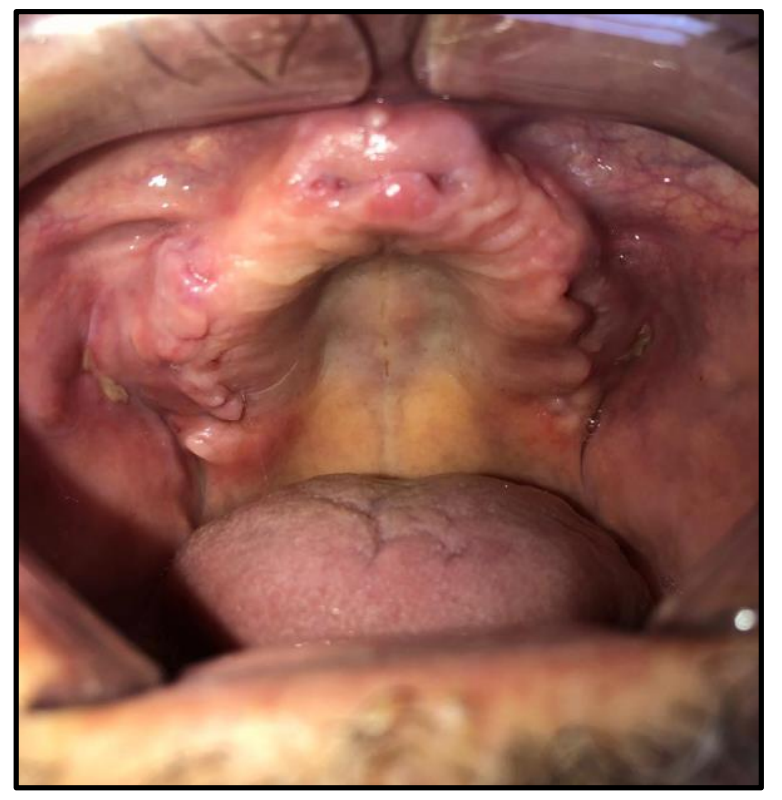

Fonte: Silva JMM, et al., 2019.

\section{DISCUSSÃO}

Para Nogueira EF, et al. (2018) e Cunha G, et al. (2017) as comunicações buco-sinusais estão associadas a intercorrências após exodontias de dentes cujas raízes apresentam estreita relação com a parede sinusal, sendo os segundos molares superiores mais acometidos, por suas raízes terem maior proximidade com o assoalho do seio maxilar.

De acordo com os autores Sanchez AS, et al., (2018) e Nogueira EF, et al. (2018) os principais fatores etiológicos incluem manobras iatrogênicas, trauma, cirurgia para remoção de cistos e enucleação de tumores, patologias ósseas, colocação de implantes e cirurgias mais complexas como a ortognática, sendo a causa mais frequente para a sua ocorrência a exodontia de um dente superior. Corroborando com o presente caso, a comunicação com o seio maxilar ocorreu após a extração do segundo molar superior direito. Com a exérese do dente, observa-se clinicamente um orifício da comunicação, e caso essa não regrida espontaneamente, 
poderá tornar-se crônica e formar uma fístula buco-sinusal, que por sua vez, causará vazamento de líquidos da boca para a cavidade nasal, epistaxe, dor, sinusite, distúrbios de fala e respiração e em casos de infecções, drenagem de secreção purulenta.

Segundo Nogueira EF, et al. (2018) as comunicações buco-sinusais são mais prevalentes em homens, de aproximadamente 40 anos de idade, concordando com o presente caso. Veras Filho RO, et al. (2015) afirma que diversas técnicas têm sido utilizadas para a resolução dessa comunicação, como o uso de retalhos mucoperiosteais, do tipo vestibular, palatino, lingual ou combinado, enxertos ósseos ou uso da bola de bichat. A conduta terapêutica para o tratamento da comunicação buco-sinusal desse caso clínico e concordando com Sanchez et al., 2018, foi realizado um retalho vestibular com a finalidade de auxiliar no fechamento da comunicação da cavidade bucal com o seio maxilar, impedindo a sua contaminação por restos alimentares e saliva provenientes da boca e posteriormente a instalação de um processo infeccioso no período pósoperatório.

Dentre as técnicas cirúrgicas, podemos destacar o uso de retalhos bucais vestibulares, o mais comum e antigo, obteve $87,2 \%$ de sucesso nos procedimentos realizados por ser bastante eficaz e feitos sob anestesia local. Essa técnica apresenta como desvantagem a diminuição da profundidade do sulco oral, o que pode interferir com a reabilitação protética, na manutenção da higiene oral, edema e dor pós-cirúrgica (SANCHEZ AS, et al., 2018; PARISE GK e TASSARA LF, 2016; NOGUEIRA EF, et al., 2018).

Coelho JR e Torre GR (2015); avaliaram por meio de tomografia computadorizada Cone Beam e classificaram as comunicações buco-sinusais em três grupos: tipo 1 com raízes penetrando no assoalho do seio, tipo $2 \mathrm{com}$ raízes em contato com o assoalho do seio e tipo $3 \mathrm{com}$ as raízes localizadas abaixo do assoalho do seio. Clinicamente observa-se um orifício da comunicação que, de acordo com o agente etiológico, varia de tamanho e radiograficamente visualiza-se uma descontinuidade da parede do seio. Os exames clínicos e radiográficos detalhados no pré-operatório são de extrema importância no planejamento cirúrgico dos casos em que há possibilidade de ocorrência da comunicação buco sinusal, esses dados permitem ao cirurgião realizar o procedimento com cautela e planejamento. Após o diagnóstico da comunicação, o profissional deve analisar o tamanho aproximado dela, visto que o tratamento está diretamente relacionado à extensão da abertura (BITTENCOURT KP, 2017).

Alguns autores afirmam que as comunicações de até $2 \mathrm{~mm}$ de diâmetro provavelmente se fecharão espontaneamente, descartando uma intervenção cirúrgica. No entanto, comunicações entre 2 e $6 \mathrm{~mm}$ ou associadas a inflamação maxilar ou periodontal, necessitam de intervenções para ajudar na manutenção do coágulo sanguíneo, uma sutura em oito deve ser feita, ou ainda associada a alguma substância hemostática dentro do alvéolo antes da sutura. Se a abertura do seio for grande ( $7 \mathrm{~mm}$ ou mais larga), deve-se considerar seu fechamento com retalho. A técnica mobiliza o tecido mole (vestibular, palatino, lingual ou combinado), enxertos ósseos ou utilização do tecido adiposo da bochecha para cobrir a abertura e promover o fechamento da comunicação (VERAS FR, 2015; PARISE GK e TASSARA LF, 2016).

Comunicações buco-sinusais maiores e não tratadas, provavelmente levarão a um quadro de sinusite aguda em quase metade dos pacientes dentro de 2 dias, e que possivelmente irão progredir até $90 \%$ dentro de 14 dias (PATEL R, et al., 2019). A comunicação buco-sinusal necessita de tratamento cirúrgico o mais precocemente possível para evitar esse tipo de complicação.

O paciente foi tratado imediatamente por meio de um retalho vestibular, apesar de Gusman DJ, et al. (2017) afirmarem que esse tipo de retalho interfere na reabilitação e manutenção da higiene protética ao proporcionar um fundo de sulco vestibular muito superficial, foi o método de escolha por propiciar uma área menos cruenta e melhor vascularização do que as técnicas supracitadas, por sua vez, reduzindo as chances de necrose e a prótese já serviu como um dispositivo de condicionamento do fundo de vestíbulo promovendo a adaptação do mesmo e diminuindo a possibilidade de redução da altura deste último.

No caso em questão, obteve-se um pós-operatório favorável e sem complicações cirúrgicas, apresentando uma boa adaptação marginal da prótese total imediata, mesmo com a dificuldade de adaptação nas primeiras semanas. 
A comunicação buco-sinusal é uma complicação relativamente frequente na área odontológica. Para o seu tratamento devem ser levados em consideração alguns parâmetros, incluindo localização, tamanho da comunicação com seio maxilar, relação com os demais dentes, altura da crista alveolar, existência de fistula e infecção sinusal, visando sempre o correto diagnóstico e tratamento a fim de evitar recidivas e obter o melhor prognóstico para o paciente.

\section{REFERÊNCIAS}

1. BITTENCOURT KP, PEREIRA JC. Comunicação Buco Sinusal, Diagnóstico e Tratamento: uma revisão de literatura. Trabalho de conclusão de curso - Centro Universitário Tiradentes, Aracaju, 2017, 13.

2. CUNHA G, et al. Comunicação buco sinusal: do manejo clínico a abordagem cirúrgica. Revista de Odontologia da UNESP, 2017; 47(34): 0-0.

3. COELHO JV, TORRE SG. Desplazamiento por iatrogenia de tercer molar a seno maxilar: reporte de caso clínico. Revista de Administração em Saúde, 2018; 75(1): 39-44.

4. GUSMAN DJR, et al. Fechamento de Fístula bucoantral: relato de caso clínico. Revista Funec Científica, 2017; 1(35): 33-45.

5. NOGUEIRA EFC, et al. Uso de Retalho Palatino Combinado com bucal no tratamento de fístula bucosinusal recidivante: relato de caso. Odontologia Clínica Científica, 2018; 17(2): 25-30.

6. PEREIRA RS, et al. A importância da cirurgia pré-protética para reabilitação de uma prótese total imediata: relato de caso. Revista da Academia Brasileira de Odontologia, 2019; 8(3): 163-141.

7. PATEL R, et al. Closure of Oro-Antral Communication Using Buccal Advancement Flap. Word J. Plast. Surg, 2019; 8(2): 262-264.

8. PARISE GK, TASSARA LF. Tratamento cirúrgico e medicamentoso das comunicações buco sinusal: uma revisão de literatura. Revista Pespectiva, 2016; 40(149): 153-162.

9. ROSA CB, et al. Fibrina rica em plaquetas e leucócitos (L-PRF). Opção de tratamento para fechamento de comunicação buco sinusal em paciente oncológico: Relato de Caso. Anais da Jornada Odontológica de Anápolis, 2019; 32(1): 171-174.

10. SANCHES A, et al. Cierre de comunicación oroantral mediante o uso de membrana de plasma: Revisão de literatura e relatório de um caso clínico. Revista de Administração em Saúde, 2018; 75(3): 153-158.

11. VERAS FR, et al. Fechamento de comunicação buco sinusal utilizando enxerto pediculado de corpo adiposo da bochecha. Revista Odonto Ciência, 2015; 25(1): 100-103. 